\title{
低能氮离子诱发丙酮与重水溶液的反应机理 *
}

\author{
石怀涁郡春林余增亮 \\ (中国科学院等离子体物理研究所, 合肥 230031)
}

\begin{abstract}
摘要 利用氮气火花放电产生离子, 其中的正离子在阴极位降的加速下 注人” 到丙酮的重水溶液中, 诱发其中 的化学反应. 利用气相色谱 - 质谱 (GC-MS) 分析离子注人后的样品, 证实有氛代产物 $\left(\mathrm{CH}_{3} \mathrm{COCH}_{2} \mathrm{D}\right)$ 、氞差基取 代产物 $\left(\mathrm{CH}_{3} \mathrm{COCH}_{2} \mathrm{OD}\right)$ 生成, 这表明低能 $\mathrm{N}^{+}$诱发重水溶液中的反应主要是由于重水分子分解产生的自由基引 起的, 其中氛自由基和氛差基自由基起重要作用; 同时, 产物中还检测到気代乙酸 $\left(\mathrm{CH}_{3} \mathrm{COOD}\right)$ 和気氨基丙酮 $\left(\mathrm{CH}_{3} \mathrm{COCH}_{2} \mathrm{ND}_{2}\right)$, 说明反应是在氧化性氛围中进行的, 氮离子俘获重水中的氞形成氞氨基可能是気氨基取代产 物生成的主要原因,也是氮 沉积” 在溶液中的重要形式。这些结果对初步揭示低能离子诱发水溶液的反应机理 具有一定的意义。
\end{abstract}

关键词：离子注人，丙酮，重水溶液，反应机理， 气相色谱 - 质谱

近年来，低能离子束辐照生物样品作为一种崭 新的辐照手段, 在农作物及微生物诱变育种、细胞 加工及基因工程等多个方面取得了重要进展 ${ }^{[1-3]}$, 但传统的注人方法必须在真空室内进行，这使低能 离子与生物样品尤其是溶液样品的作用受到限制． 我们利用气体常压火花放电产生低能离子, 研究其 与水溶液样品的相互作用 ${ }^{[4-5]}$. 研究表明低能离子 诱发溶液体系中的反应与固态的离子注人具有明 显不同的特点. 由于溶液良好的流动性, 形成的损 伤产物能够很快地扩散开去, 不会被后来的离子损 伤; 另外, 溶液环境有利于损伤碎片的进一步化合 及一些中间产物的保留, 因而, 相对固态注人而言, 溶液中的反应更为充分，是诱发化学反应的有效途 径, 但目前对这类反应的机理尚不清楚。本文利用 火花放电产生的低能氮离子注人丙酮的重水溶液, 对反应机理进行了初步的探讨.

\section{1 实验部分}

\section{1 材料}

丙酮为上海溶剂厂产品, 分析纯, 蒸馏两次后 使用; 重水 $\left(\mathrm{D}_{2} \mathrm{O}\right)$ 为北京化学试剂总厂产品, 纯度 $(w)>99.99 \%$. 为降低污染, 所有玻璃仪器在使用 前均于 $500{ }^{\circ} \mathrm{C}$ 烘烤 $4 \mathrm{~h}$.

\section{2 方法}

1.2.1 低能离子的注入

为了模拟研究低能离子与水溶液的相互作用, 我们设计了图 1 所示实验装置。在图 1 中, $\mathrm{A}$ 为电

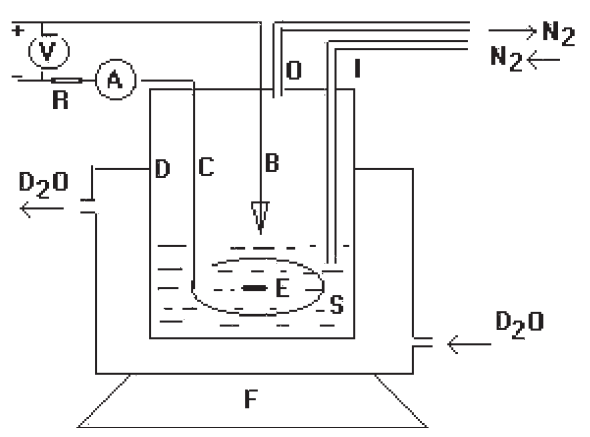

图 1 低能氮离子与重水溶液的相互作用模拟装置图

Fig. 1 Apparatus for reaction in aqueous solution induced by low energy $\mathrm{N}^{+}$ions

A: amperemeter; B: anode; C: cathode; D: glass container;

E: magnet; F: magnetic stirrer; I: gas inlet; O: gas outlet;

R: resistor; S: solution sample

2001-04-19 收到初稿, 2001-07-04 收到修改稿.联系人：石怀彬（E-mail：shihuaibin@ sina. com）。*国家自然科学基金资助项目 (29772033) 


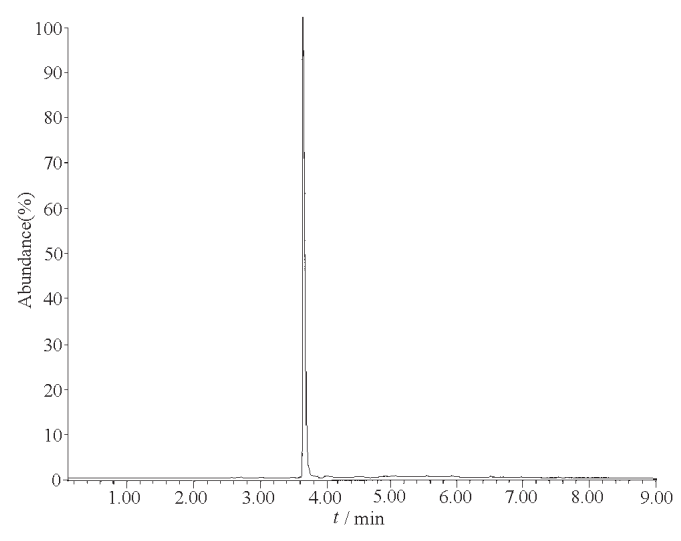

图 2 对照样气相色谱分离图

Fig. 2 GC chromatogram of compare sample

流表, B 为针状不锈钢阳极, 距液面 $0.5 \sim 1 \mathrm{~mm}$, $C$ 为银丝, $R$ 为限流电阻, 阻值 $450 \mathrm{k} \Omega$. 当阴阳极 之间的电位差达到一定值后，其间的气体将电离 产生等离子体，其中的阳离子在阳极与水面的鞘 层中得到加速后进人水溶液, 诱发水溶液中的化 学反应 ${ }^{[6]}$.

取 $8 \mathrm{~mL}$ 重水加人放电室 (即图 1D) 中, 加 2 $\mathrm{mL}$ 丙酮, 振荡、摇匀后取出少量溶液作为对照. 向放电室内通人高纯氮（> 99. 99\%）5～10 min 后, 接通电源, 输人电压调到 $3 \mathrm{kV}$, 在放电时电流 稳定在 $6 \mathrm{~mA}$. 适时取出少量反应液, 留待分析.

1. 2.2 试样 GC-MS 分析

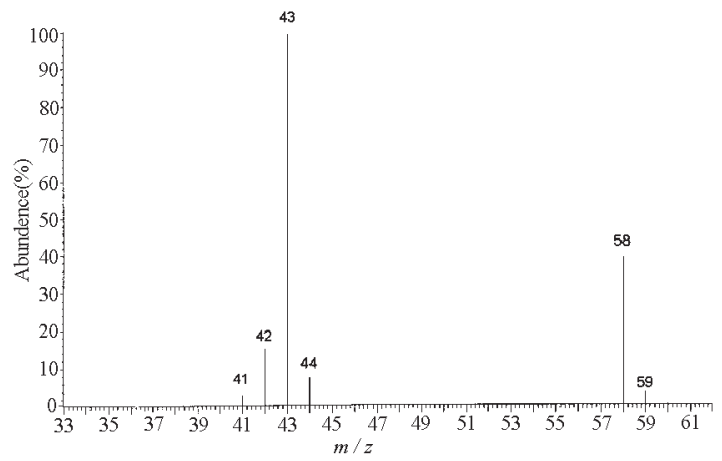

图 3 图 2 中 $3.75 \mathrm{~min}$ 峰质谱图

Fig. 3 Mass spectrum of the peak at $3.75 \mathrm{~min}$ in Fig. 2

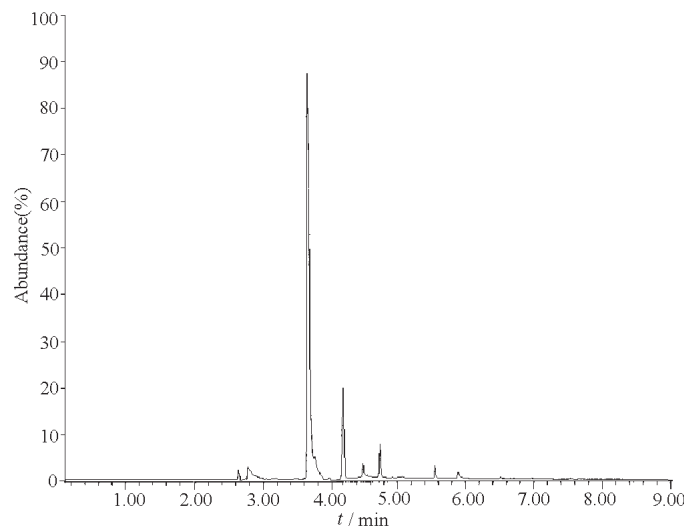

图 4 低能 $\mathbf{N}^{+}$注入丙酮重水溶液 $2 \mathrm{~h}$ 样品气相色谱分离图

Fig. 4 GC chromatogram of acetone solution with $D_{2} \mathbf{O}$ as solvent implanted by low energy $\mathrm{N}^{+}$for $2 \mathrm{~h}$

样品及对照在美国 PERKIN-ELMER 公司 Auto System XL 型毛细管 $(30 \mathrm{~m} \times 0.25 \mathrm{mmi} . \mathrm{d} . \times 0.25$ $\mu \mathrm{mdf})$ 气相色谱仪及 Tuto-Mass 质谱仪上进行 GC-MS 分析, 升温区间为 $40 \sim 120{ }^{\circ} \mathrm{C}$, 每分钟 $20{ }^{\circ} \mathrm{C}$ 升至 $120{ }^{\circ} \mathrm{C}$,再保温 $6 \mathrm{~min}$, EI 离子化能量 $70 \mathrm{eV}$.

\section{2 结果与讨论}

对照样品的 GC-MS 分析结果, 如图 2 所示, 图 3 为对应于图 2 中各峰的化合物的质谱图.

由图 2 可见, 原料中丙酮和重水纯度很高, 气相 色谱图中几乎 看不出” 任何明显的杂质. 图 3 对应 的化合物应为丙酮, 从该图可知重氢交换反应产生的

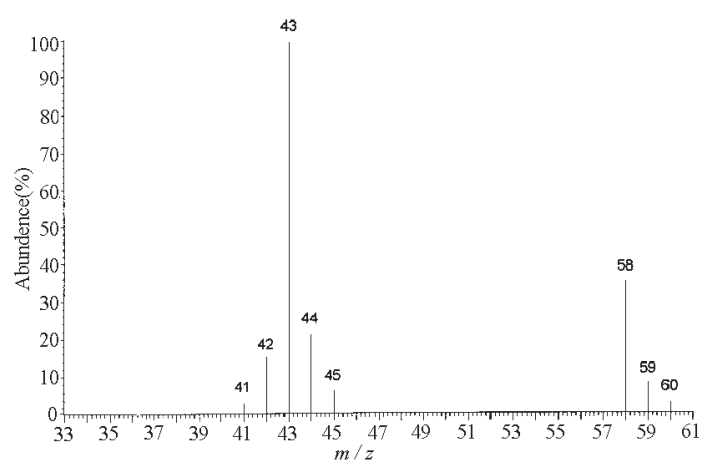

图 5 图 4 中 $3.75 \mathrm{~min}$ 峰质谱图

Fig. 5 Mass spectrum of the peak at $3.75 \mathrm{~min}$ in Fig. 4 


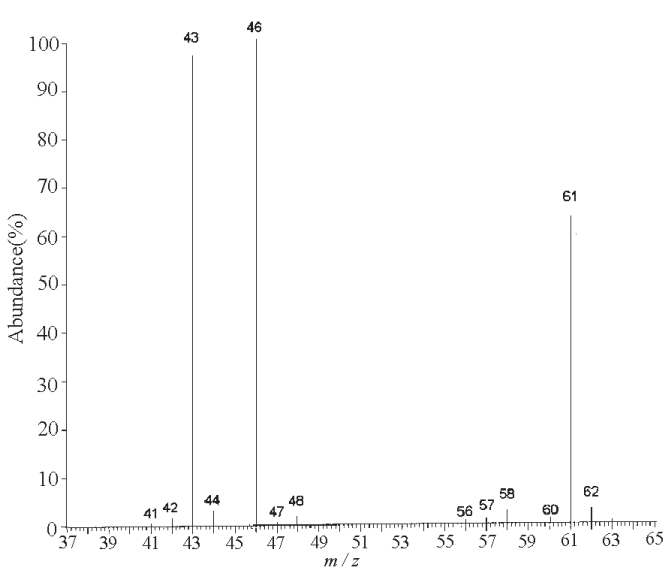

图 6 图 4 中 $4.20 \mathrm{~min}$ 质谱图

Fig. 6 Mass spectrum of the peak at $4.20 \mathrm{~min}$ in Fig. 4

氛代丙酮很少, 图中看不出.

样品分析结果如图 4 图 8 所示. 图 4 为样品 气相色谱分离图, 图 5 图 8 为对应于图 4中各峰 的化合物的质谱图, 相应化合物可能的结构式及质 谱图的简单解析亦于图下标明 ${ }^{[7,8]}$.

在图 5 中, 由于存在明显的 $m / z 59, \mathrm{~m} / z 44$ 两个峰, 故认为图 4 中 $3.75 \mathrm{~min}$ 峰应为

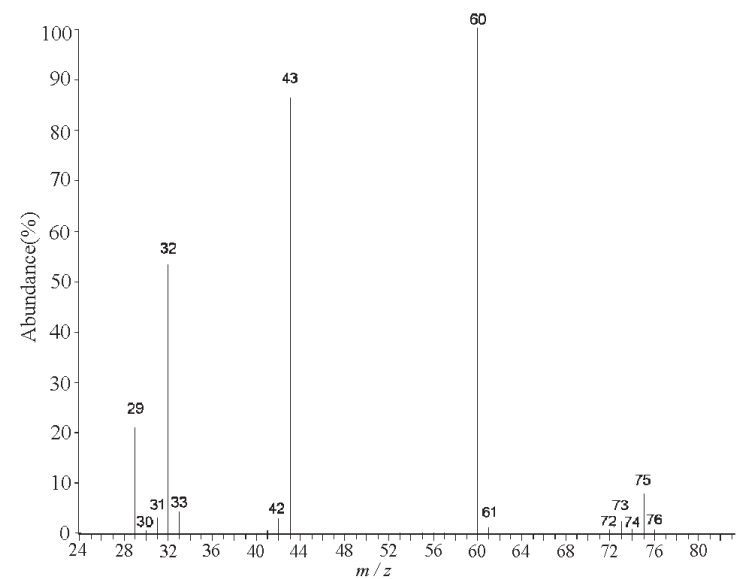

图 7 图 4 中 $4.55 \mathrm{~min}$ 峰质谱图

Fig. 7 Mass spectrum of the peak at $4.55 \mathrm{~min}$ in Fig. 4
$\mathrm{CH}_{3} \mathrm{COCH}_{3}$ 与 $\mathrm{CH}_{3} \mathrm{COCH}_{2} \mathrm{D}$ 的混合物 :
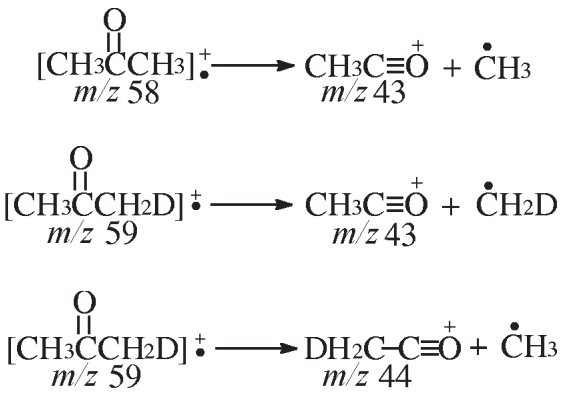

由图 6 可知，该化合物可能的结构式为 $\mathrm{CH}_{3} \mathrm{COOD}$ :
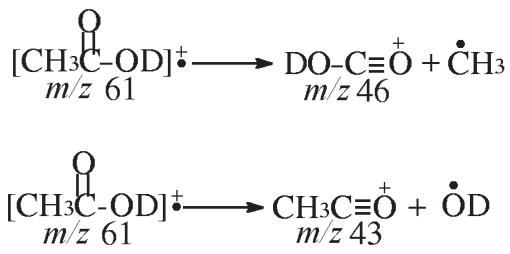

图 7 中的化合物可能为 $\mathrm{CH}_{3} \mathrm{COCH}_{2} \mathrm{ND}_{2}$, 其中主 要碎片的产生如下:
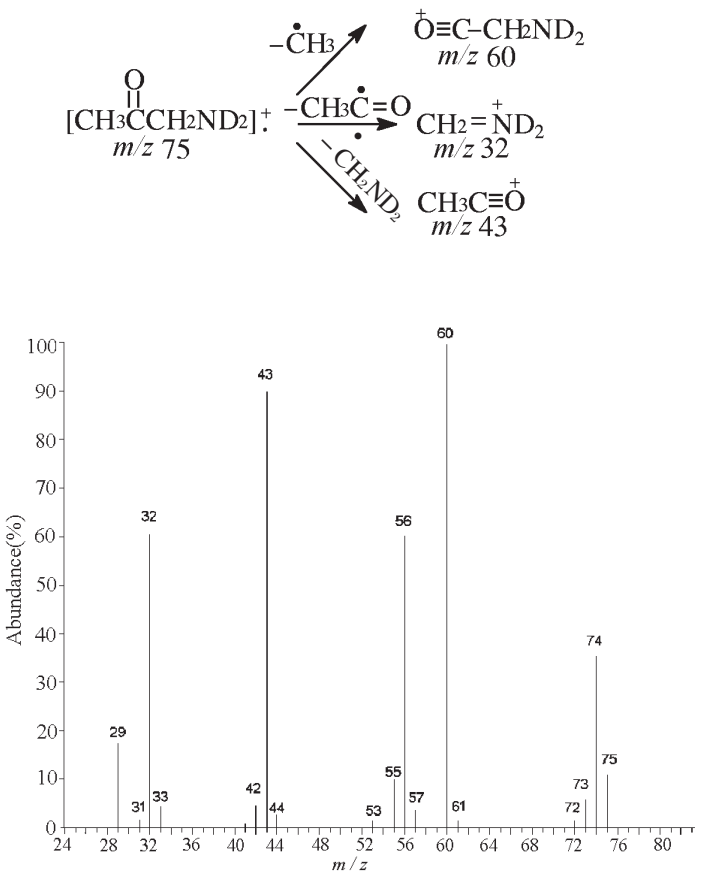

图 8 图 4 中 $4.74 \mathrm{~min}$ 峰质谱图

Fig. 8 Mass spectrum of the peak at $4.74 \mathrm{~min}$ in Fig. 4 
依据图 8 及反应原料, 我们认为该化合物可能 为 $\mathrm{CH}_{3} \mathrm{COCH}_{2} \mathrm{OD}$, 其中主要碎片的产生过程如下:

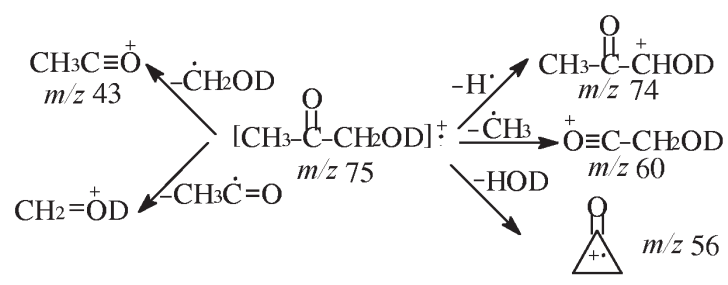

通过以上分析可以看出, $\mathrm{N}^{+}$注人丙酮重水 溶液后有気代、氞羟基、氞氨基取代产物及氞代 乙酸生成, 由于溶剂是 $\mathrm{D}_{2} \mathrm{O}$, 故 $\mathrm{CH}_{3} \mathrm{COCH}_{2} \mathrm{D}$ 、 $\mathrm{CH}_{3} \mathrm{COCH}_{2} \mathrm{OD}$ 的生成可以认为是因 $\mathrm{N}^{+}$注人导致 重水分子分解的结果 ${ }^{[9]}$ :

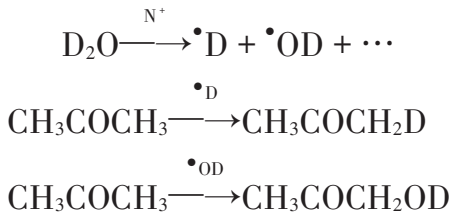

即重水分子分解产生的自由基是诱发反应的首要 因素，其中氛自由基和氛羟基自由基起主要作用. $\mathrm{CH}_{3} \mathrm{COCH}_{2}-\mathrm{ND}_{2}$ 的生成可能是 $\mathrm{N}^{+}$俘获 $\mathrm{D}_{2} \mathrm{O}$ 中的 $\mathrm{D}$ 生成 $\cdot \mathrm{ND}_{2}$, 继而取代丙酮中 $\alpha-\mathrm{H}$ 的结果 ${ }^{[10]}$ :

$$
\begin{gathered}
\mathrm{D}_{2} \mathrm{O} \longrightarrow \stackrel{\mathrm{N}^{+}}{\longrightarrow} \cdot \mathrm{D}+{ }^{\cdot} \mathrm{OD}+\cdots \\
\mathrm{N}^{+}+{ }^{\cdot} \mathrm{D} \longrightarrow{ }^{\longrightarrow} \mathrm{ND} \longrightarrow \\
\stackrel{\mathrm{ND}_{2} \longrightarrow \mathrm{CH}_{3} \longrightarrow \mathrm{COCH}_{3}}{\longrightarrow} \mathrm{CH}_{3} \mathrm{COCH}_{2}-\mathrm{ND}_{2}
\end{gathered}
$$

由此可见，氨基取代产物并非 $\mathrm{N}^{+}$与溶质直接作用 的结果，而是通过溶剂分子的间接作用形成的，这 种氞氨基取代产物可能是外来氮 沉积” 在溶液中 的重要形式, 但这种 质量沉积” 效应与固态 $\mathrm{N}^{+}$注 人时靶原子的移位、重组造成的沉积效应显然是不 同的 ${ }^{[11-12]} \cdot \mathrm{CH}_{3} \mathrm{COOD}$ 的生成机理目前尚不清楚, 我们认为可能与重水分解产生的 ${ }^{\circ} \mathrm{OD}$ 有关:

$$
\begin{aligned}
& \mathrm{CH}_{3} \mathrm{COCH}_{3} \stackrel{\bullet \mathrm{OD}}{\rightarrow} \mathrm{CH}_{3} \mathrm{COCH}_{2} \mathrm{OD} \stackrel{\bullet \text { OD }}{\rightarrow} \\
& \mathrm{CH}_{3} \mathrm{COCOOD} \underset{[0]}{-\mathrm{CO}_{2}} \mathrm{CH}_{3} \mathrm{COOD}
\end{aligned}
$$

可见 $\mathrm{N}^{+}$诱发重水溶液中的反应不仅仅是自由 基取代反应, 还涉及碳链的氧化降解, 而这种氧化 作用可能与重水分解产生的気差基自由基有密切
关系

总之, 低能 $\mathrm{N}^{+}$注人重水溶液中的反应主要是 由于重水分子分解产生的気自由基和気羟基自由 基引起的，这些自由基进攻溶质分子形成产物；另 一方面, $\mathrm{N}^{+}$可以与氞自由基化合, 形成氞氨基自由 基, 继而与溶质分子作用,形成氞氨基取代产物 ; 同 时, 可能由于重水分子分解产生大量気差基自由 基，使体系氧化性增强，最终导致溶质分子氧化降 解，但对这种氧化作用的根本原因目前尚不能肯 定,有待进一步的研究.

\section{References}

1 Yu ZL, Wu YJ, Deng JG, He JJ, Huo YP. Nucl. Instr. Meth. Phys. Res., 1991, B59/60: 705

2 Shao CL, Yu ZL. Nuclear Techniques, 1997, 20(7): 423 [邵春林,余增亮。核技术 ( He JiShu), 1997, 20 (7)：423]

3 Yang JB, Wu LJ, Wu JD, Wu YJ. Chinese Sci. Bull., 1994, 39(16)：1530 [杨剑波, 吴李君, 吴家道, 吴跃进。科学通报 (Kexue Tongbao), 1994, 39(16): 1530]

4 Han JW, Yu ZL. Spectroscopy and Spectral Analysis, 1999, 19 (1): 19 [韩建伟, 余增亮. 光谱学与光谱分析 (Guangpuxue yи Guangpu Fenxi), 1999, 19(1): 19]

5 Han JW, Lu C, Yu ZL. J. Radiat. Res. Radiat. Process. 1998, 16 (2): 89 [韩建伟, 卢 超, 余增亮. 辐射研究与辐射工艺学 报 (Fushe Yanjiu yu Fushe Gongyi Xuebao), 1998, 16(2): 89]

6 Jian YF. Handbook of Staticelectricity, Trans. Group of Handbook of Staticelectricity. Beijing: Science Press, 1981: 3 [静电 手册. 静电手册翻译组译. 北京: 科学出版社, 1981：3]

7 Yan CT. Foundation of the Analysis of Organic Compounds. Beijing: High Education Press, 1991：153 [阎长泰. 有机分析 基础. 北京:高等教育出版社, 1991：153]

8 Wang YJ, Song ZF. Spectral Analysis and Chromatographic Analysis. Beijing: Peking University Press, 1995: 86 [王彦吉, 宋增福. 光谱分析与色谱分析. 北京: 北京大学出版社, 1995: 86]

9 Zhang MW. Induction to Radiation Chemistry. Hefei: USTC Press, 1993: 84 [张曼维. 辐射化学入门. 合肥: 中国科大出 版社, 1993: 84]

10 Kutcher G J, Green A E S. Radiation Research, 1976, 67: 408

11 Shao C L, Yu Z L. Radiat. Phys. Chem., 1997, 49(3): 337 12 Yu Z L. IEEE Transactions on Plasma Science, 2000, 28 (1): 128 


\title{
Mechanism of the Reaction between Low Energy $\mathrm{N}^{+}$and $\mathrm{CH}_{3} \mathrm{COCH}_{3}$ with $\mathrm{D}_{2} \mathrm{O}$ as Solvent*
}

\author{
Shi Huai-Bin Shao Chun-Lin Yu Zeng-Liang \\ ( Institute of Plasma Physics, Chinese Academy of Sciences, Hefei 230031)
}

\begin{abstract}
Low energy ions were produced by $\mathrm{N}_{2}$ glow-discharge. The positive ones were accelerated into acetone solution with $\mathrm{D}_{2} \mathrm{O}$ as solvent to induce chemical reactions. GC-MS analysis showed that $\mathrm{CH}_{3} \mathrm{COCH}_{2} \mathrm{D}$, $\mathrm{CH}_{3} \mathrm{COCH}_{2} \mathrm{OD}$ were produced by such kind of implantation. Thus, it was proved that the reaction was mainly caused by radicals generated by decomposition of water molecules, $\mathrm{D}^{\bullet}$ and ${ }^{\circ} \mathrm{OD}$ played an important role in the process. Meanwhile, $\mathrm{CH}_{3} \mathrm{COOD}$ and $\mathrm{CH}_{3} \mathrm{COCH}_{2} \mathrm{ND}_{2}$ were also found in the products, so it was concluded that the reaction was carried out under an oxidative atmosphere. The capture of $\mathrm{D}$ from $\mathrm{D}_{2} \mathrm{O}$ by $\mathrm{N}^{+}$to form ${ }^{\circ} \mathrm{ND}_{2}$ radicals was not only an initial step to produce $\mathrm{CH}_{3} \mathrm{COCH}_{2} \mathrm{ND}_{2}$ but also served as a probable pattern for "nitrogen deposition". All these were helpful to reveal the mechanism of the reaction induced by low energy $\mathrm{N}^{+}$implanting into solution samples.
\end{abstract}

Keywords: Ion implantation, Acetone, $\mathrm{D}_{2} \mathrm{O}$ solution, Mechanism, GC-MS 